\title{
Social Stairs: taking the Piano Staircase towards long- term behavioral change
}

\author{
Michel Peeters ${ }^{1}$, Carl Megens ${ }^{1}$, Elise van den Hoven $^{12}$, Caroline Hummels ${ }^{1}$, Aarnout \\ Brombacher ${ }^{1}$ \\ ${ }^{1}$ Faculty of Industrial Design, Eindhoven University of Technology \\ Den Dolech 2, 5612AZ Eindhoven, The Netherlands \\ \{m.m.r.peeters, c.j.p.g.megens, c.c.m.hummels, a.c.brombacher\}@tue.nI \\ ${ }^{2}$ Design, Architecture \& Building, University of Technology Sydney \\ PO Box 123, Broadway NSW 2007, Australia \\ elise.vandenhoven@uts.edu.au
}

\begin{abstract}
This paper addresses the development of Social Stairs, an intelligent musical staircase to change people's behavior in the long-term to take the stairs in favor of the elevator. Through designing with the Experiential Design Landscape (EDL) method, a design opportunity was found that social engagement encouraged people to take the stairs at work in favor of the elevator. To encourage this social behavior, people who involved each other and worked together whilst using the Social Stairs were treated with more diverse orchestral chimes that echoed up the stairwell. In this paper we reflect on the differences between the persuasive system of the well-known Piano Staircase and the Social Stairs. We report on the deployment of the Social Stairs for a period of three weeks in the public space within the university community and identify opportunities for triggering intrinsic motivation, social engagement and how to keep people involved in the long-term.
\end{abstract}

Keywords: wellbeing, persuasion, active lifestyle, social engagement, longterm behavioral change, Experiential Design Landscapes, Experiential Probes

\section{Introduction}

Modern societal trends and technologies enable us to live our lives with comfort and fun. While these developments create quality of life, with respect to our health and well-being there are downsides as well. In our current society we are dealing with an increasing sedentary and inactive lifestyle with all the consequences (e.g. obesity, diabetes) that entail for our health and well-being (Schroeder, 2007). Persuasive technology is often used and applied to provide solutions, to persuade people to become more active again. One of the pinnacle examples of persuasive technology is the Piano Staircase (Volkswagen, 2009)(Figure 1), a design concept of a musical staircase which was built next to the escalator at the Odenplan subway station in Stockholm, Sweden. People could choose to either use the stairs or the escala- 
tor, with the latter being the more popular but unhealthier choice. The designers of the Piano Staircase decided to try to change people's behavior by transforming the stairs into a giant piano keyboard. By applying pressure on each step the staircase would play a musical note. The resulting video of the Piano Staircase shows commuters discovering the Piano Staircase, getting curious and triggered towards the behavior of staircase by playing with the steps and eventually using the stairs more often than the escalator. Through the interactive steps the staircase successfully persuaded and motivated $66 \%$ more people to use the stairs instead of the escalator throughout the day they shot the video (Volkswagen, 2009). The Piano Staircase is persuasive technology designed with an explicit intention to change people's attitude or behavior to choose the stairs over the escalator (IJsselsteijn et al., 2006)(Lockton et al. 2010). It is clearly shows the potential of how technology can persuade people, by triggering new motivations, to change an attitude, belief or behavior through persuasion and social influence, not through coercion (Fogg 2002).

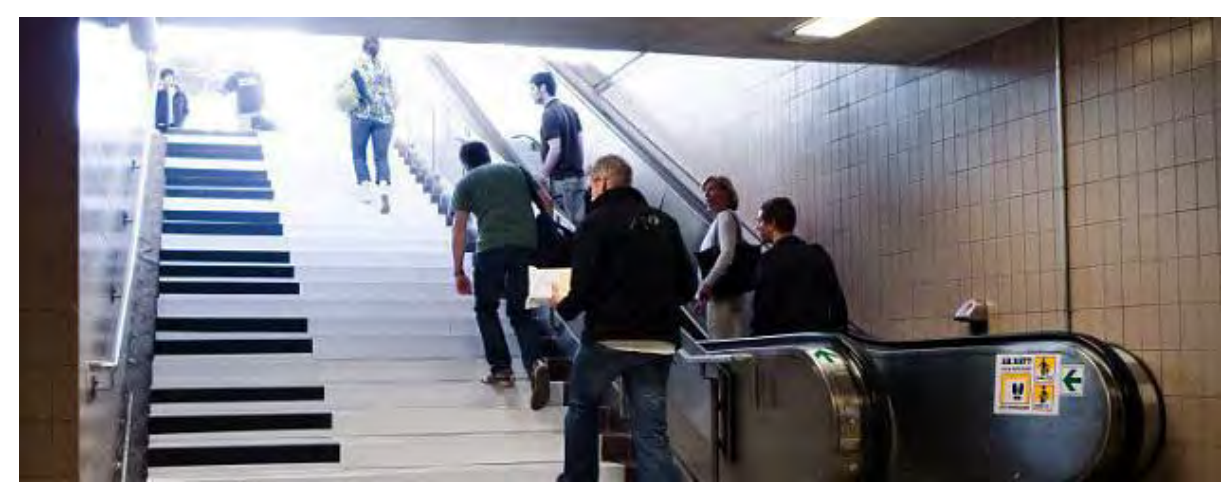

Fig. 1. Piano Staircase; applying pressure on each step plays a musical note.

For the case of designing for wellbeing towards a more active and healthy lifestyle we fully support the attempts and believes of the Persuasive Technology field that behavioral change is favorable for a healthy society. We question however whether the current examples like the Piano Staircase will lead to long-term structural behavioral change. The Piano Staircase really made a difference on the first day, the moment people got introduced to this new concept and it raised their curiosity and motivation. What is not shown in the video is what happens when the same person passes the stairs every day. Will he/she still be triggered by the Piano Staircase in the long run to play with it and actually use the stairs instead of the escalator? Could this persuasion lead to a long-term intrinsic motivation for people to keep using the stairs?

\section{Designing for behavioral change in the long-term}

BJ Fogg's (2009) behavior Model describes three elements that must converge at the same moment for a behavior to occur: Motivation, Ability, and Trigger. When behavior change does not occur, at least one of those three elements is missing. 
IJsselsteijn et al. (2006) point out that changing human behavior typically takes a long time and that there are, to their knowledge, "hardly any user studies available that have looked at the long-term effectiveness of persuasive technology". To explore long-term behavioral change through design we did a longitudinal experiment. For this we developed and used an Experiential Design Landscape (EDL) (Gent et al. 2011), an infrastructure in neighborhoods where all stakeholders work together, creating experienceable propositions together with them, which evolve over time.

These propositions, Experiential Probes (EPs), are intelligent, open, sensor-enhanced, networked products-service systems that enable people to develop new and emerging behavior and, in parallel, enable detailed analysis of the emerging data patterns by researchers and designers as a source of inspiration for the development of future systems, products and services. We were interested in how design can evoke emerging or changing behavior that will lead to people's intrinsic motivation in the longterm to take the stairs in favor of the elevator. For this purpose we created an EDL called Social Stairs, where we developed and evaluated several Experiential Probes over time.

\subsection{Social Stairs}

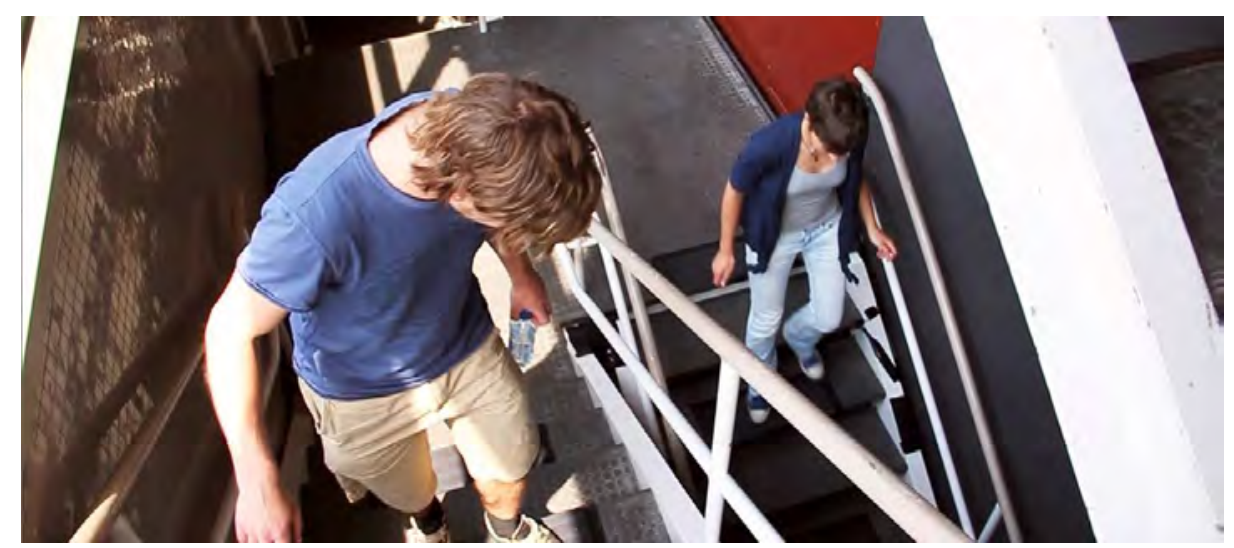

Fig. 2. People working working together in the Social Stairs EDL.

In the university's main building, sensors and sound actuators were installed to the main staircase as well as a camera to observe the EDL (see figure 2). The overall goal was to get people to walk past the elevator and use the stairs instead. At first ascending and descending sounds were mapped to the steps, similar to the Piano Staircase, but not directly mimicking a piano. This, similar as on the Piano Staircase, triggered people to play with the steps, walking over the stairs in different speeds and just randomly stepping and jumping on them. The sound echoed past the elevator and raised awareness, triggering people to take a look. In contradiction to the Piano Staircase the choice between the elevator and stairs is not presented equally, as the stairs are positioned behind the elevator. In early experiments it was found that social engagement encouraged more active behavior. One of the noticeable occurrences was that a lot of 
people played together with the staircase, trying to create sounds or even music together. After a few days the designers saw that people were inviting peers to join them on the stairs, helping to promote the staircase to a larger audience. As the design is not final in an EDL, it is treated as a probe, several alterations or complete new attempts can be made by the designers to steer or find the behavior they envisioned. In this case the designers wanted to encourage this social behavior. People who worked together whilst using the Social Stairs were treated with a richer louder, orchestral chime that echoed up the stairwell. This resulted in a richer dynamic experience, the stairs sounded different depending on the situation the people were in.

\subsection{Behavior on Social Stairs}

Social Stairs provided the designers with user data of three weeks (i.e. log data of steps, interviews and video) which was utilized to continuously do design iterations but also to analyze and test whether the intended effects were actually met. Through the data (Figure 3) they got insight in different types of behavior. For instance, people invited others to join them at the Social Stairs and create a soundscape together. Other people were actively seeking opportunities to create a joined soundscape, by patiently waiting for a while in the stairwell. Unexpected and emergent behavior also occurred; some people were meeting up in the stairwell on a daily basis, similar to a hangout, and formed medium to large groups (2-10 people) to create a gigantic joined soundscape. Others got to know new people through the Social Stairs.

What the designers found particularly interesting was the example of some recurring visitors who were driven by curiosity about possible new types of behavioral layers of the Social Stairs. They explained to the designers that they actually hadn't made a deliberate decision to take the stairs over the elevator but they were just curious if the Social Stairs was still working and if there was something going on in the stairwell. Initially they were driven by curiosity but taking the stairs also became a new habit for them. It's even questionable if they were still deciding between the stairs and the elevator, by choosing the stairs in favor of the elevator.

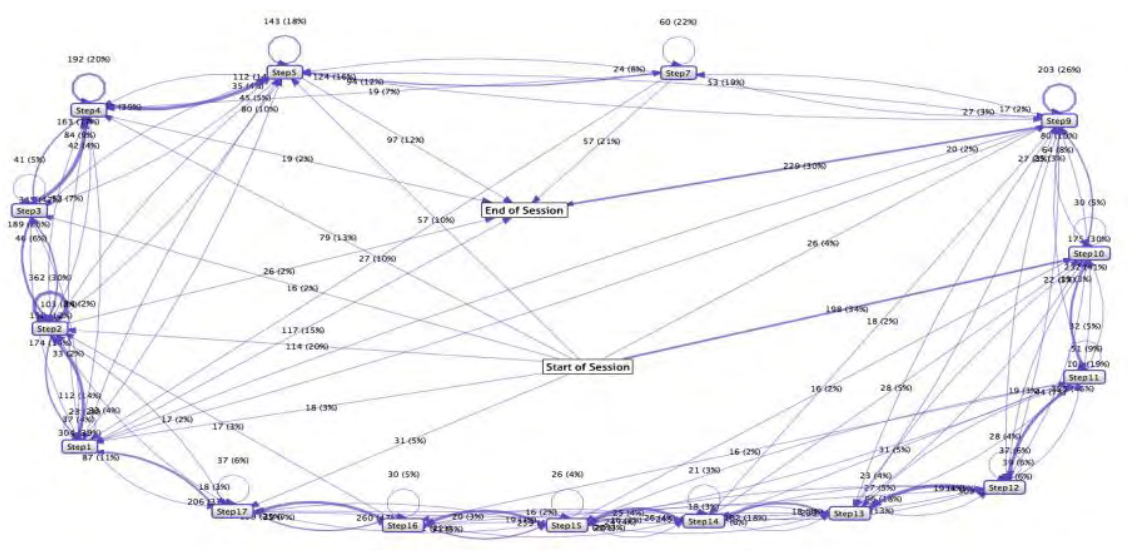


Fig. 3. Through these visualizations insight can be gained in the amount of playfulness that occurred that day. Low sequential steps and more loops means people played around and jumped around in a non-sequential order. The visualization is made in such a way that it for instance becomes easy to compare different days to each other, showing the triggered playfulness of different Experiential Probes.

\section{Probing towards intrinsic motivation to take the stairs}

When we look at BJ Fogg's (2009) behavior Model, the Social Stairs changed very little to people's 'ability' to physically climb the stairs or their ability to choose the stairs in favor of the elevator. The two other important elements for a behavior to occur i.e. 'trigger' and 'motivation' however did change (still with the understanding that both elements have to take place at the same moment together with people's ability, for an eventual behavior to occur). Social Stairs was a result of an EDL in which the triggers were 'open scripts' which could evolve over time; they were adapted or sometimes even completely (re)designed based on the log data of the Social Stairs as well as video.With the Social Stairs we wanted to design and probe towards triggering people's intrinsic motivation to take the stairs.

With intrinsic motivation we would like to refer to the work of White (1959) and Deci (1975) where intrinsic motivation refers to motivation to enact a behavior for its inherent satisfaction, in alignment with one's personal values or attitudes, and not for a separable external consequence. Moreover, intrinsic motivation seems to increase the likelihood of the behavior being performed (Deci, 1975) and seems to lead to sustained behavior (Deci, 1975). With EDLs we therefore also aim to design towards sustainable behavioral change through triggering people's intrinsic motivation. As people may lose attention to the act of persuasion we therefore believe that true sustainable behavioral change most likely has to come from within people themselves to last. Therefore, designers should focus their designs to help people trigger intrinsic motivation towards changing behavior, not persuade and extrinsically motivate them to act or behave different in that moment of time. It is however very hard to predict which trigger will lead to intrinsic motivation on the long term. For designers it is almost impossible to predict what behavior will emerge or change once people start to use their designs. By using the EDL method designers can probe and find out during the process of design.

\section{Discussion}

The EDL method allows designers to explore and play around with their designs and peoples' resulting behavior. At this moment the Social Stairs EDL is still ongoing and much is still left to explore. The designers are further developing and exploring ways to make the sounds more dynamic. Several probes are created in which people over time can change the sounds, both the character of the sound itself, but also through 
repeating loops of recent visitors creating an echo-effect. Aim is to create a multilayered experience, in which people can continuously (and jointly) explore and deepen the interaction with the Social Stairs. This way the designers aim to design for long-term structural new behavior change instead of 'just' renewing the sounds of the Social Stairs. Next to this, research has to be done on the data outcome of the stairs. In the first three weeks of the EDL a lot of data has been accumulated which needs further and deeper analysis. Already an analysis of playful behavior has been done (Figure 3), but we believe there is more understanding of behavior to be gained from the combination of the log data, video and interviews. Nevertheless the EDL method shows to be promising for long term, structural behavior change. Through the combination of continuously analyzing and designing in the EDL designers can react and anticipate on (unexpected) behavior of the people involved.

\section{Acknowledgements.}

This work is being carried out as part of the "Design for Wellbeing" project, sponsored by the Dutch Ministry of Economic Affairs under the IOP-IPCR program. We would like to thank the students involved in this project, responsible for the design of the probes: Nadine van Amersvoort, Rhys Duindam, Nick Hermans, Max Sakovich \& Bart Wolfs

\section{References}

1. Deci EL (1975) Intrinsic motivation. Plenum, New York

2. Fogg, B.J.: Persuasive technologies: Introduction. Communications of the ACM 42 (1999)

3. Fogg, B.J.: Persuasive Technology: Using Computers to Change What We Think and Do. Morgan Kaufmann Publishers, San Francisco (2002)

4. Fogg, B.J.: A behavior model for persuasive design. Proceedings of the 4th International Conference on Persuasive Technology.

5. Gent, S.H. van, Megens, C.J.P.G., Peeters, M.M.R., Hummels, C.C.M., Lu, Y. \& Brombacher, A.C. (2011). Experiential design landscapes as a design tool for market research of disruptive intelligent systems. Proceedings of the 1st Cambridge Academic Design Management Conference. Cambridge: University of Cambridge.

6. IJsselsteijn, W.A., de Kort, Y.A.W., Midden, C., Eggen, B., \& van den Hoven, E. (eds., 2006). Persuasive Technology. Proceedings of the First International Conference on Persuasive Technology for Human Well-Being. Lecture Notes in Computer Science, vol. 3962. Berlin: Springer

7. Lockton, D., Harrison, D., and Stanton, N. : The Design with Intent Method: A design tool for influencing user behavior. Applied Ergonomics 41, 3 (2010), 382- 392.

8. Schroeder, S.: We Can Do Better - Improving the health of the American people. The New England Journal of Medicine, vol. 357, pp. 1221-1228. (2007)

9. Volkswagen Group Sverige AB 2009, Sodertalje, accessed 21 January 2013 $<$ http://www.thefuntheory.com/piano-staircase $>$

10. White, R.W. (1959) Motivation reconsidered. Psychol Rev 66:297-333 